\title{
Heat Flux of a Transferred Arc Driven by a Transverse Magnetic Field
}

\author{
Naomi Matsumoto, Takashi Usami, Ikumi Kuno, Takeo Yamamoto, \\ Masaya Sugimoto, Seiji Kumagai, and Koichi Takeda
}

\author{
Department of Machine Intelligence and System Engineering, Akita Prefectural University, \\ Yurihonjo 015-0055, Japan \\ Correspondence should be addressed to Naomi Matsumoto, m11a016@akita-pu.ac.jp \\ Received 12 August 2009; Accepted 21 September 2009 \\ Recommended by Jainagesh Sekhar
}

Theoretical consideration of a magnetically driven arc was performed to elucidate the variation of heat flux with an imposed DC magnetic field. Experiments were conducted to confirm the validity of the theoretical model. The heat flux decreased concomitantly with increased imposed magnetic flux density. Theoretical predictions agreed with experimental results.

Copyright (C) 2009 Naomi Matsumoto et al. This is an open access article distributed under the Creative Commons Attribution License, which permits unrestricted use, distribution, and reproduction in any medium, provided the original work is properly cited.

\section{Introduction}

Because of intense energy concentration to a small arcroot, transferred arcs have been widely used for cutting and welding of metals. However, conventional arcs remain inconvenient for heating and melting over a wide area. Many attempts have been made to expand arc profiles using external magnetic fields [1]. Takeda developed a magnetically driven arc by imposing an alternating magnetic field perpendicular to a transferred arc $[2,3]$. In the oscillatory motion of such a magnetically driven arc, the amplitude increases with the increase of the imposed magnetic flux density. The oscillating arc can be regarded as a broad heat source with oscillation amplitude width, as presented in Figure 1. The heat flux distribution is known to be nonuniform along the oscillatory motion of the arc [4]. For practical applications of such magnetically driven arcs, a clear understanding of the nonuniformity in the heat flux is necessary.

In this work, the variation of the heat flux in the arc root was studied under a DC magnetic field imposed perpendicularly to the arc.

\section{Theoretical Considerations}

2.1. Displacement of an Arc-Root with Imposed Magnetic Flux Density. A schematic arrangement of the magnetically driven arc and a coordinate system used in the present work are presented in Figure 2.

The plasma torch is located on the $z$-axis. The anode expands in the $x-y$ plane. An external DC magnetic field is imposed in the direction of $x$. Without an imposed magnetic field, the arc-root center position is located at the origin $(0,0,0)$. The arc is assumed to be cylindrically symmetric to the $z$-axis. The interaction of the arc current and the external magnetic field engenders arc movement in the $y$ direction. Displacement of the arc-root center position ( 0 , $Y, 0)$ increases with increased the imposed magnetic flux density. [3]:

The following relation was reported in a previous work

$$
\frac{Y}{L}=R-\sqrt{R^{2}-1} .
$$




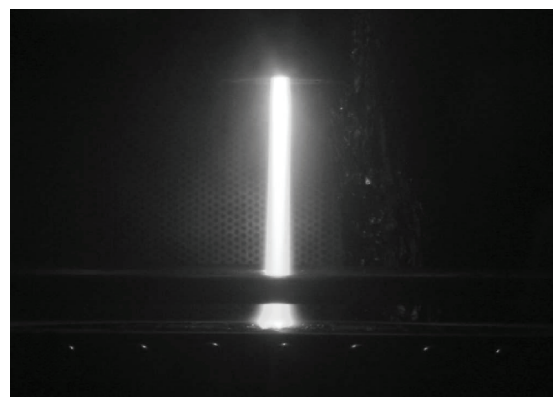

(a)

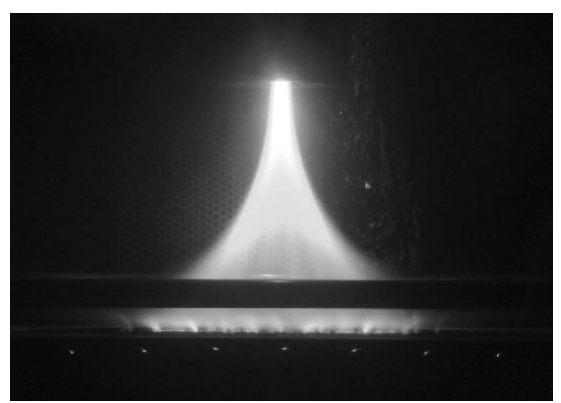

(b)

FIgURE 1: Typical plasma arc without magnetic field (a), and magnetically driven arc in an alternating magnetic field (b).

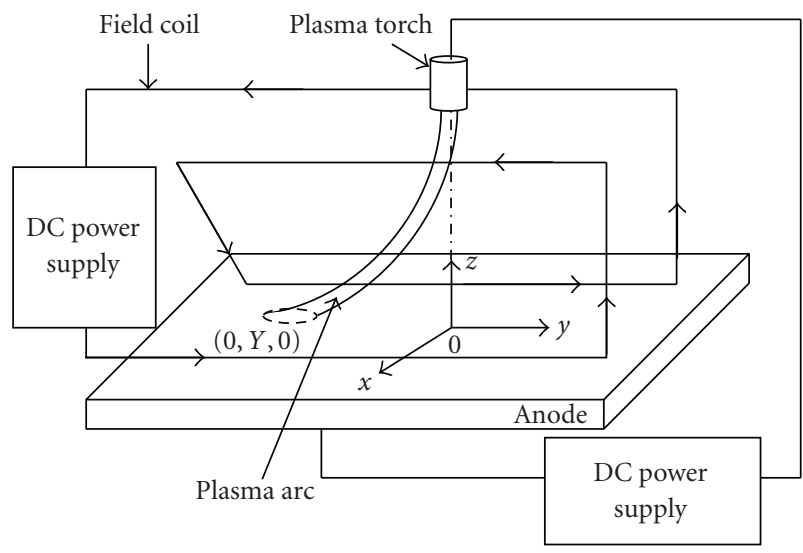

FIGURE 2: Experimental setup for a magnetically driven arc.

Therein, $Y, L$, and $R$, respectively, represent the arc-root center position, the standoff distance between the torch and the anode, and the radius of curvature, which varied inversely with magnetic flux density.

2.2. Arc-Root Deformation Caused by the Imposed Magnetic Field. As portrayed in Figure 3, the arc-root profile under no magnetic field is assumed as a circle of diameter $d_{o}$. When the magnetic field is imposed, the arc-root shape deforms from a circle to an ellipse with major diameter $d_{Y}$ and minor diameter $d_{0}$.

Considering the relation of (1), the ratio of the major diameter to the minor diameter in the arc-root is expressed as

$$
\frac{d_{y}}{d_{o}}=\frac{1+(Y / L)^{2}}{1-(Y / L)^{2}} .
$$

Here, we introduce $h_{Y}(x, y)$ as the heat flux distribution function in the arc-root centered at $Y$. As described in the experimental results, the heat flow from the arc to the anode remains constant, independent of the imposed magnetic flux density. Therefore, the following ratio of the heat flux is assumed:

$$
\frac{h_{Y}(0, Y)}{h_{0}(0,0)}=\frac{1-(Y / L)^{2}}{1+(Y / L)^{2}} \text {. }
$$

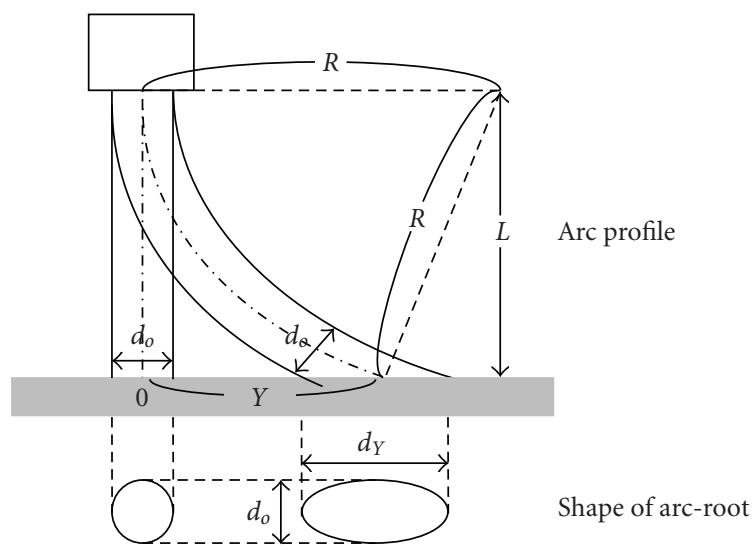

FIgURE 3: Schematic illustration on the arc root deformation.

In that equation, $h_{0}(0,0)$ and $h_{Y}(0, Y)$, respectively, represent the heat flux of the arc-root center at $Y=0$ and that at $Y$, according to the definition of $h_{Y}(x, y)$.

\subsection{Assumption of the One-Dimensional Heat Flux Distri-} bution Function. To simplify the consideration, the twodimensional heat source of the arc is transformed into a one-dimensional heat source; the arc-root is assumed as a line heat source with length $d_{Y}$, instead of an elliptic heat source with major diameter $d_{Y}$ and minor diameter $d_{o}$. The one-dimensional heat flux is expected to be satisfied by the following condition:

$$
H_{Y}(y)=\int_{-\infty}^{\infty} h_{Y}(x, y) d x
$$

The one-dimensional distribution function of the heat flux under no magnetic field is expressed as $H_{0}(y)$ because the arc-root center is located at $Y=0$. Considering the experimental observation that the heat flux of the arc has a maximum value at the center of the arc-root and decreases its value with distance from the center [5], the following 


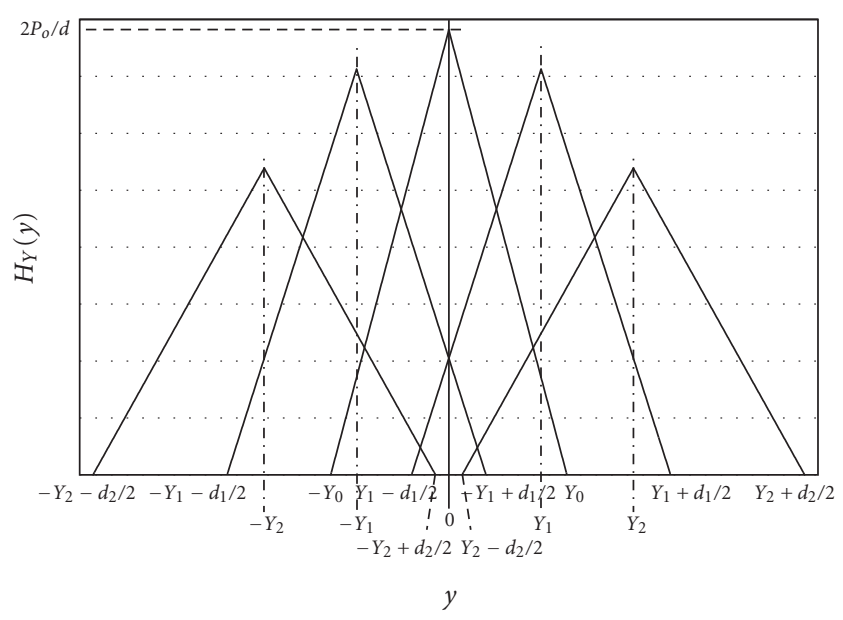

FIgURE 4: Variation of $H(y)$ with $Y$.

assumption is adopted in the present work for the onedimensional distribution function:

$$
\begin{aligned}
& H_{0}(y)=\frac{2 P_{o}}{d_{0}}\left\{1-\left|\frac{2 y}{d_{0}}\right|\right\} \quad \text { for } 0 \leq|y|<\frac{d_{Y}}{2}, \\
& H_{Y}(y)=0 \text { for }|y| \geq \frac{d_{Y}}{2} .
\end{aligned}
$$

Therein, $P_{o}$ represents the total power transferred from the arc to the anode. Under the imposed magnetic field, the distribution function for the heat flux centered on $y=Y$ is expressed as shown below:

$$
\begin{aligned}
& H_{Y}(y)=\frac{2 P_{o}}{d_{Y}}\left\{1-\left|\frac{2(y-Y)}{d_{Y}}\right|\right\} \text { for } 0 \leq|y-Y|<\frac{d_{Y}}{2}, \\
& H_{Y}(y)=0 \text { for }|y-Y| \geq \frac{d_{Y}}{2} .
\end{aligned}
$$

In those equations, $d_{Y}$ denotes the one-dimensional heat source length, which varies with $Y$ according to the relation of (2). Schematic distributions of the heat flux for various $Y$ are depicted in Figure 4. As clarified there, the peak value of the heat flux decreases and the arc-root length increases concomitantly with increased $Y$ (or increased $B$ ). The validity of the rough simplification is examined through comparison of theoretical predictions with experimental results.

2.4. Heat Flow to a Calorimeter. To estimate the heat flux, a calorimetric measurement is often used, as described in the next section. The heat flux is not measured directly using the calorimeter. According to a schematic illustration of a calorimeter depicted in Figure 5, the heat flow to the calorimeter, $W_{Y}\left(y^{*}\right)$ should be given as the following relation:

$$
W_{Y}\left(y^{*}\right)=\int_{y^{*}-l}^{y^{*}+l} H_{Y}(y) d y .
$$

Therein, in Figure 5, the center of the calorimeter with width of $2 l$ is at $y=y^{*}$. Furthermore, subscript $Y$ in (8) signifies the center position of the arc-root.

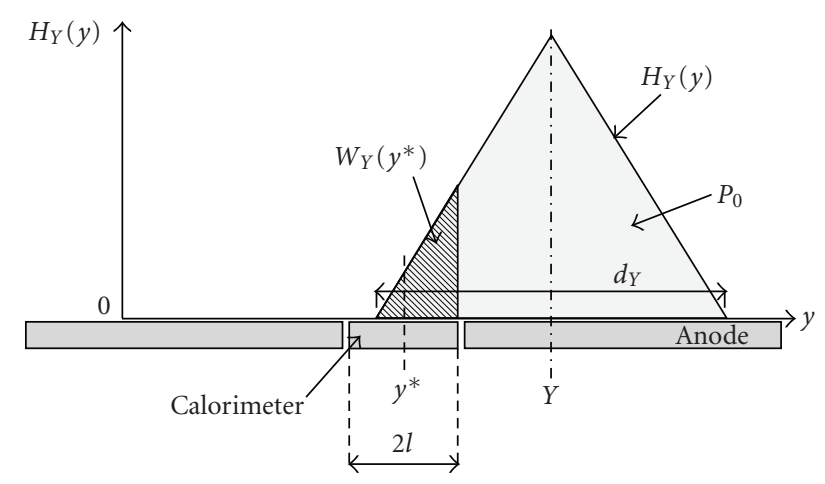

FIGURE 5: Schematic illustration of the heat flow from the arc to a calorimeter.

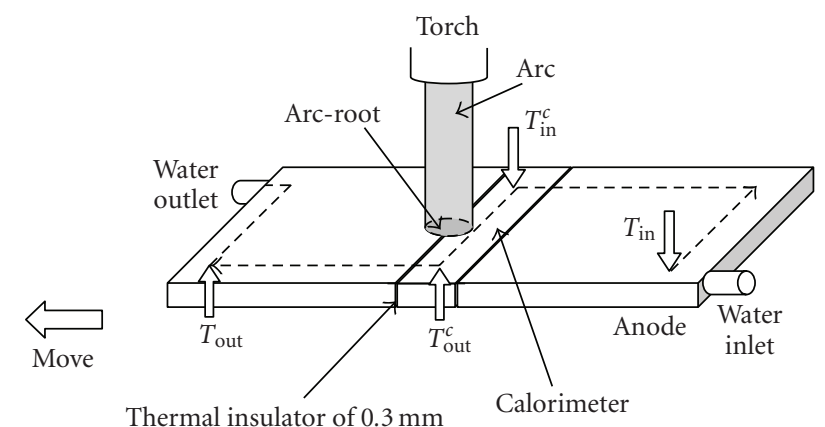

Figure 6: Anode assembly and calorimeter to measure the heat flow.

$$
\begin{aligned}
& \text { At } y^{*}=0 \text { and } Y=0 \text {, } \\
& \qquad W_{0}(0)=\frac{4 l\left(d_{o}-l\right)}{d_{o}{ }^{2}} P_{o} .
\end{aligned}
$$

The diameter of the arc-root under no magnetic field is determined using (9), as

$$
d_{o}=\frac{2 P_{o} l+\sqrt{4 P_{o}^{2} l^{2}-4 P_{o} W_{0}(0)}}{W_{0}(0)} .
$$

Then, using $d_{0}, H_{Y}(y)$ is obtainable from (6) and (2). Finally, $W_{Y}\left(y^{*}\right)$ is calculable theoretically from (8) using $H_{Y}(y)$.

\section{Experiments}

Experiments were conducted to confirm the validity of the theoretical consideration. A schematic depiction of the experimental arrangement is presented in Figure 2. Experimental conditions are similar to those described by Yamamoto et al. [4]. A transferred arc was produced between a plasma torch with a tungsten cathode and a water-cooled copper anode. The standoff distance between the torch orifice and the anode was fixed at $70 \mathrm{~mm}$. A power supply connected to the torch and the anode was operated in a constant current mode. The arc current was fixed at $130 \mathrm{~A}$. Argon gas was fed to the torch as a plasma-forming gas at the flow rate of $4.5 \times 10^{-4} \mathrm{~kg} / \mathrm{s}$. An external magnetic field perpendicular to the arc was generated using DC 


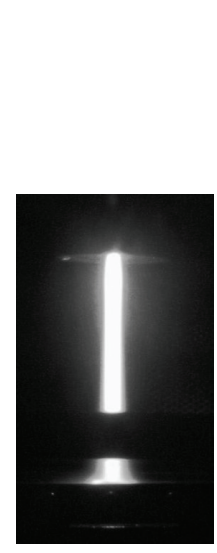

(a)

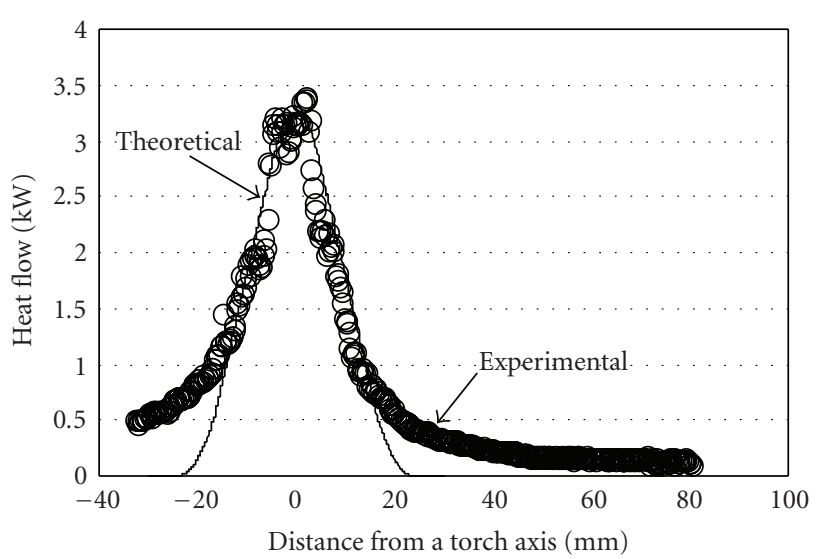

(b)

FIgURE 7: Comparison of experimental variation of heat flow to the calorimeter with the theoretical one under no magnetic field $(Y=0)$.

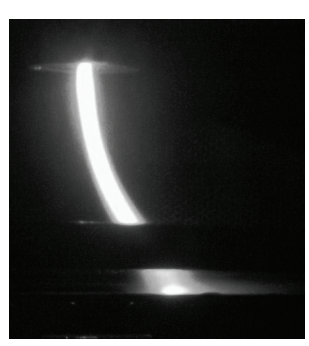

(a)

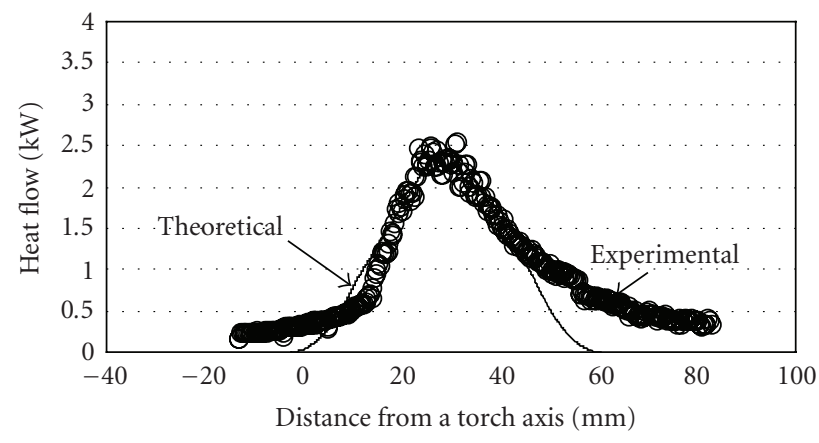

(b)

Figure 8: Comparison of the experimental variation of heat flow from a magnetically driven arc to the calorimeter with the theoretical variation of heat flow at $Y=2.9$.

electric current through a two-turn rectangular field coil. The maximum magnetic flux density in the coil center was $2.22 \mathrm{mT}$ at the coil current of $300 \mathrm{~A}$.

In this study, instead of the heat flux distribution, we measured the variation of the heat flow using an assembly of a specially designed water-cooled copper anode block, as depicted in Figure 6. Designated as a calorimeter hereinafter, it was $10 \mathrm{~mm}$ wide, $10 \mathrm{~mm}$ thick, and $100 \mathrm{~mm}$ long. The calorimeter moved in the direction of the arc movement at $0.33 \mathrm{~mm} / \mathrm{s}$ using its motor drive mechanism. The heat flow from the arc to the calorimeter was estimated according to the temperature increase of the cooling water through this block.

\section{Comparison of Theoretical Predictions with Experimental Results}

4.1. Determination of $P_{o}$ and $d_{o}$. For numerical calculations, it is necessary to determine unknown parameters $P_{o}$ and $d_{o}$. From the measurement of temperature difference between input temperature $T_{\text {in }}$ and output temperature $T_{\text {out }}, P_{o}$ was obtained as

$$
P_{o}=7.2 \mathrm{~kW} .
$$

It was confirmed that $P_{o}$ was independent of the imposed magnetic flux density. The heat flow at $y^{*}=0$ under no magnetic field was also measured and $W_{0}(0)$ of $3.3 \mathrm{~kW}$ was obtained. Substituting $P_{0}, l$, and $W_{0}(0)$ into (10), the arc diameter $d_{o}$ was determined as

$$
d_{o}=38.4 \mathrm{~mm} .
$$

4.2. Comparison of Theoretical Predictions with Experimental Results. Variations of the heat flow from the arc were measured by changing the calorimeter position in two different cases. For the case of no imposed magnetic field, the arc profile and the variation of the heat flow transferred to the calorimeter with $y^{*}$ are portrayed, respectively, in Figures $7(\mathrm{a})$ and $7(\mathrm{~b})$.

In the other case, the arc was deformed by the imposed magnetic field generated by the field coil current of $160 \mathrm{~A}$, as presented in Figure 8(a). The center position of the arcroot moved from $Y=0$ to $Y=29 \mathrm{~mm}$ : the obtained variation of the heat flow to the calorimeter with $y^{*}$ is presented in Figure 8(b). Using experimental values of $P_{o}$ and $d_{o}$ obtained in Section 4.1, numerical calculations of $W_{Y}\left(y^{*}\right)$ were conducted for the two cases of $Y=0$ and $Y=29 \mathrm{~mm}$. The theoretical variation for $Y=0$ and that 
for $Y=29 \mathrm{~mm}$ are shown, respectively, in Figures 7(b) and $8(\mathrm{~b})$.

The agreement of theoretical predictions with experimental results is good despite the rough assumptions used for theoretical considerations.

\section{Conclusions}

The variation of heat flux from an arc with magnetic flux density was considered theoretically under a DC magnetic field imposed perpendicular to the arc. Two-dimensional heat flow was transformed into one-dimensional heat flow to simplify the theoretical model. The distribution function of the heat flux in the arc-root was assumed as triangular.

Theoretical considerations yielded these predictions.

(1) The arc root on the anode expands with the increase of the imposed magnetic flux density.

(2) The heat flux density decreases with increase of the magnetic flux density.

Experiments were conducted to measure the heat flux distribution using a specially designed calorimeter. The comparison of experimental results with theoretical predictions confirmed the validity of the assumptions used in the theoretical model.

\section{References}

[1] J. E. Harry and D. Goodwin, "Surface heat treatment using a plasma torch with a magnetically traversed arc," in Proceedings of the 4th International Conference on Advances in Welding Processes, vol. 181, Harrogate, UK, May 1978.

[2] K. Takeda, "Oscillating plasma arc by external AC magnetic field," in Proceedings of the International Workshop on Plasma Jets in the Development of New Materials Technology, pp. 485492, Frunze, Russia, September 1990.

[3] K. Takeda, "Generation of magnetically oscillating plasma arc and its properties," Journal of High Temperature Society, vol. 16, p. 357, 1990.

[4] T. Yamamoto, K. Takeda, T. Toh, and J. Tanaka, "Distribution of heat flux transported by a magnetically driven arc," Thin Solid Films, vol. 515, no. 9, pp. 4228-4233, 2007.

[5] M. Tanaka, H. Terasaki, R. Narita, K. Kobayashi, H. Fujii, and M. Ushio, "Anode heat transfer in TIG arc and its effect on weld penetration," Quarterly Journal of the Japan Welding Society, vol. 23, no. 3, pp. 398-404, 2005. 

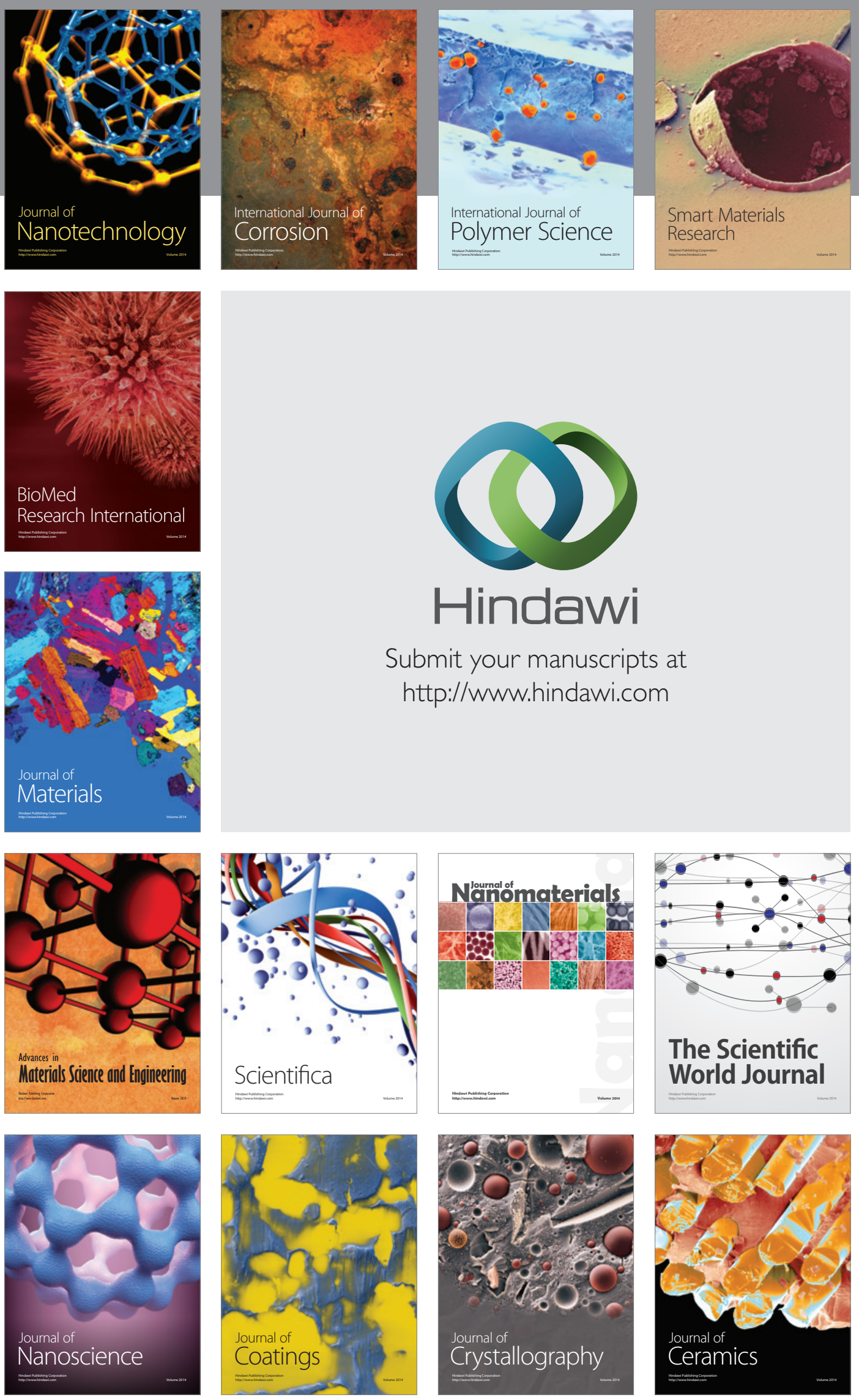

The Scientific World Journal

Submit your manuscripts at

http://www.hindawi.com

\section{World Journal}

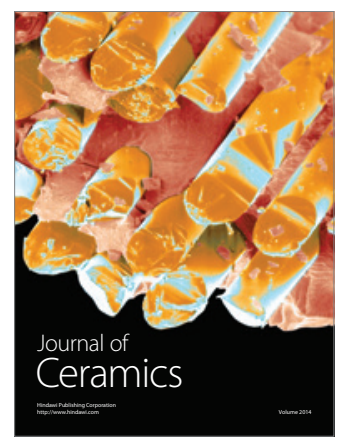

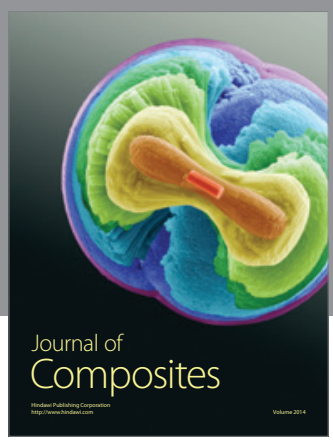
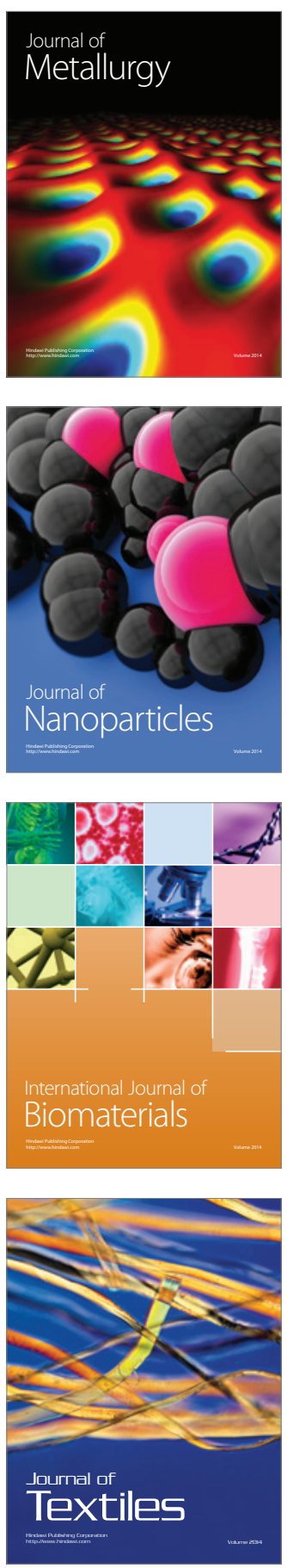